\title{
Malária em Grávidas de uma Maternidade Pública de Rio Branco (Acre, Brasill)
}

\author{
Malaria in Pregnant Women of a Public Maternity \\ of Rio Branco (Acre State, Brazil)
}

Regina Jarude, Ronald Trindade, José Tavares-Neto

\begin{abstract}
RESUM0
Objetivo: descrever a freqüencia dos achados clinico-laboratoriais em grávidas portadoras de malária.

Métodos: foi realizado estudo descritivo, série de casos, de 445 grávidas da Maternidade e Clinica de Mulheres Bárbara Heliodora (Rio Branco, Acre), do periodo de janeiro de 1996 a dezembro de 2001, com diagnóstico parasitológico de malária. Foram revistos os prontuários do periodo e selecionados os casos portadores de malária.

Resultados: no total de pacientes ( $n=33.420$ ) internadas no periodo, estavam incluidas 445 (1,4\%) grávidas. Entre essas, a freqüência da infecção por Plasmodium vivax foi de $52,8 \%(n=235)$, por P. falciparum, de 43,8\% (n=195), e 3,4\% $(n=15)$ por ambos os plasmódios. As alterações clínico-laboratoriais mais freqüentes $(p<0,05)$ foram observadas nas portadoras do P. falciparum: mucosas descoradas, ictericia, diminuição da hemoglobina e hematócrito, hipoglicemia e elevação dos niveis séricos das aminotransferases, uréia, creatinina e das bilirrubinas. Somente houve um caso de óbito (1/445) em gestante com P. falciparum. Quanto ao concepto, foram registrados: abortos $(1,3 \%)$, prematuridade $(1,1 \%)$ e baixo peso ao nascer $(1,1 \%)$.

Conclusões: esses achados refletem a repercussão da malária durante a gestação, bem como justificam que as unidades de obstetricia, especialmente da região amazônica, tenham serviços capacitados para o diagnóstico e tratamento desses casos.
\end{abstract}

PALAVRAS-CHAVE: Complicações da gravidez. Infecções. Malária. Abortamento. Anemia.

\section{Introdução}

A malária é um dos grandes problemas de Saúde Pública porque os plasmódios são encontrados em áreas onde habita quase a metade da população mundial. Nas Américas, 21 países são endêmicos e 357 milhões $(38,6 \%)$ de habitantes estão expostos ao risco de adoecer. O Brasil é responsável por um terço dos casos notificados de malária ${ }^{1}$. A quase totalidade dos casos procede da

Maternidade e Clínica de Mulheres Bárbara Heliodora Rio Branco, Acre

Correspondência:

Regina Jarude

Conjunto Tropical I, Quadra D, Casa 45

69910-480 - Rio Branco - AC

e-mail: jarude@uol.com.br
Amazônia Legal, onde prevalecem características ambientais altamente favoráveis à permanência dos plasmódios, principalmente porque há bons criadouros naturais do anofelino-vetor ${ }^{2}$. Por isto, essa região do país contém áreas de alto e médio risco de infecção malárica ${ }^{2}$, estando o Estado do Acre entre as áreas de médio risco ${ }^{3}$.

Dessa forma, na maior parte do território brasileiro, a malária é responsável pelos menores indicadores de saúde, notadamente entre a população infantil, de grávidas e naqueles procedentes de região indene $e^{4,5}$. Nas grávidas, a imunodepressão associada ao período gestacional ${ }^{6}$ e aquela decorrente da multiplicação do plasmódio na placenta ${ }^{7-9}$ concorrem, juntamente com outros fatores (anemia ferropriva, deficiências dos serviços de pré-natal, etc.), para a exacerbação das 
manifestações e das complicações clínicas, bem como dos efeitos sobre o concepto ${ }^{6,10,11}$.

É freqüente a associação da malária durante o período gestacional com amplo espectro de complicações, como hipoglicemia grave, insuficiência renal aguda, coagulação intravascular disseminada, quadro cerebral, entre outras ${ }^{4}$, ou como causa de abortamento, prematuridade ou de recém-nascido de baixo peso ${ }^{6,11-14}$. Em conseqüência, a malária na grávida deve ser considerada potencialmente grave, especialmente se a mulher for primigesta ${ }^{14}$, o agente etiológico for $o P$. falciparum $^{12,14}$ e/ou quando a mulher não tiver história de malária prévia ${ }^{13}$.

A maternidade-clinica de mulheres, referência na cidade de Rio Branco (Estado do Acre), mantém serviço capacitado para o diagnóstico e terapêutica de casos de malária. Porém, desta região não há casuísticas conhecidas que permitam melhor avaliar o impacto da malária sobre as grávidas. Por isto, foi planejado este estudo visando conhecer as características demográficas, epidemiológicas, clínico-laboratoriais e terapêuticas utilizadas nas grávidas atendidas nos últimos seis anos.

\section{Pacientes e Métodos}

Esta série de casos foi levantada no Serviço de Arquivo Médico e Estatística da Maternidade e Clínica de Mulheres Bárbara Heliodora, MCMBH (Rio Branco, Acre), onde um dos autores (RJ), desde junho de 1986, é responsável pelos casos clínicos internados nessa unidade de referência da Secretaria de Estado de Saúde e Saneamento do Estado do Acre. Também na cidade de Rio Branco, a MCMBH é a unidade de referência para as pacientes de alto risco do Sistema Único de Saúde e onde há serviço neonatal de terapia intensiva. Por isto, atende não só à grande demanda espontânea dos municipios do Vale do Acre (com 11 sedes municipais e aproximadamente $64,9 \%$ da população total do Estado, calculada em 2001 em 557.527 mil habitantes ${ }^{15}$ ), bem como às grávidas de alto risco da região do Vale do Juruá (abrangendo os outros 11 municipios do Estado do Acre ${ }^{15}$ ) e aos casos procedentes das regiões da Extrema (Estado de Rondônia) e da Boca do Acre (Estado do Amazonas).

O levantamento abrangeu o período de seis anos, de $1^{\circ}$ de janeiro de 1996 a 31 de dezembro de 2001 , correspondendo a 33.420 prontuários médicos revistos. Inicialmente, foram incluídos os prontuários que continham registro de pesquisa de Plasmodium sp ou o seu resultado. Em seguida, os números de registro dos prontuários foram confrontados com os registros dos livros do Laborató- rio de Patologia Clínica da $\mathrm{MCMBH}$ e, em caso de divergência, foram comparados aos registros do Setor de Admissão, do Serviço Social e/ou do Setor de Enfermagem. Somente foram incluídos os casos com diagnóstico parasitológico de malária, determinado pelo teste da gota espessa ${ }^{2}$, e desde que a espécie do plasmódio tivesse sido avaliada no esfregaço do sangue periférico ${ }^{2}$. Após essa seleção, somente foram incluídos os casos com diagnóstico clínico e/ou ultra-sonográfico da gravidez e avaliação da semana gestacional (conforme o período trimestral da gestação).

Dos casos selecionados, os dados de prontuário foram registrados em ficha individual, com informações sobre características demográficas (idade, cidade onde reside e se da área rural ou urbana), clínico-laboratoriais à admissão e da terapêutica aplicada. Nos exames de laboratório, os limites inferiores de normalidade considerados foram: $11 \mathrm{~g} / \mathrm{dL}$ de hemoglobina, $36 \%$ de hematócrito, glicose $60 \mathrm{mg} /$ $\mathrm{dL}$, uréia $15 \mathrm{mg} / \mathrm{dL}$, creatinina $1,4 \mathrm{mg} / \mathrm{dL}$, bilirrubina total $1,2 \mathrm{mg} / \mathrm{dL}$, bilirrubina indireta 0,9 $\mathrm{mg} / \mathrm{dL}$, aspartato aminotransferase (AST) $4 \mathrm{U} / \mathrm{L}$, e alanina aminotransferase (ALT) $4 \mathrm{U} / \mathrm{L}$.

Posteriormente, as informações coletadas foram inseridas na planilha Excel e analisadas pelo "software" SPSS ${ }^{\circledR}$ (versão 10.0). As variáveis qualitativas foram estudadas primeiro através das suas proporções e, quando indicado, pelo teste do $\chi^{2}$ ou o teste exato de Fisher. Caso a variável fosse quantitativa, contínua, foram estimadas as médias e desvios-padrão, e, havendo a necessidade de comparar dois grupos, foi aplicado o teste $t$ de Student. Nessas análises estatísticas, a diferença foi considerada significante se a probabilidade (p) do erro tipo I fosse $\leq 5 \%(\mathrm{p} \leq 0,05)$.

Este trabalho foi aprovado pelo comitê de ética em pesquisa da Fundação Hospital Estadual do Acre.

\section{Resultados}

Entre os 33.420 prontuários do período de estudo, foram selecionados $496(1,4 \%)$ prontuários de grávidas com diagnóstico parasitológico de malária, sendo analisados $445(89,7 \%)$ casos. Entre os 51 casos excluídos, não houve determinação da espécie do plasmódio em $8(15,6 \%)$ e de 43 pacientes $(84,3 \%)$ faltavam os dados obstétricos (diagnóstico da gestação e/ou da semana gestacional).

Entre as 445 grávidas incluídas, a média da idade foi 23,1 $( \pm 6,3)$ anos com limites de 12 e 49 anos. No conjunto, as espécies do plasmódio tiveram a seguinte distribuição: $52,8 \% P$. vivax $(\mathrm{n}=235)$, $43,8 \%$ P. falciparum $(\mathrm{n}=195)$ e a forma mista $(P$. vivax $+P$. falciparum $)$ correspondendo a $3,4 \%(n=15)$. 
Nesses três grupos, as médias das idades das grávidas foram, respectivamente, 23,2 $( \pm 6,3), 22,9$ ( \pm $6,1)$ e $24,0( \pm 8,2)$ anos, mas, ao serem comparadas entre si, não houve diferença significante $(p>0,79)$. Em nenhuma paciente foi diagnosticado o P. malariae.

Nas análises subseqüentes, os 15 casos com a forma mista foram excluídos e os casos de $P$. vivax $(\mathrm{n}=235)$ foram comparados aos de $P$. falciparum $(\mathrm{n}=195)$. Na Tabela 1, a distribuição dos casos conforme a faixa etária das pacientes ficou próxima ao limite de significância estatística $(p>0,05)$, porque houve redução significativa $(\mathrm{p}<0,04)$ de casos com $P$. falciparum na faixa etária de 28 a 35 anos de idade. A mesma Tabela 1 mostra o predomínio $(\mathrm{p}<0,03)$ de grávidas procedentes da área rural do município de Rio Branco.

Tabela 1 - Faixa etária, procedência e período gestacional das grávidas com malária, segundo a espécie do plasmódio.

\begin{tabular}{cccc} 
& \multicolumn{3}{c}{ Espécie do plasmódio, $\mathbf{n}(\%)$} \\
& $\boldsymbol{P}$. vivax & $\boldsymbol{P}$. falciparum & $\mathbf{p}^{\mathbf{a}}$ \\
\hline Faixa Etária $(\mathrm{n})$ & $(235)$ & $(195)$ & $>0,05$ \\
$12-19$ & $74( \pm 31,5)$ & $55( \pm 28,2)$ & \\
$20-27$ & $101( \pm 43,0)$ & $95( \pm 48,7)$ & \\
$28-35$ & $47( \pm 20,0)$ & $25( \pm 12,8)$ & \\
$\geq 36$ & $13( \pm 5,5)$ & $20( \pm 10,3)$ & \\
Procedênciab & & & \\
Rio Branco $(\mathrm{n})$ & $(167)$ & $(139)$ & $<0,03$ \\
$\quad$ Urbana & $74( \pm 44,3)$ & $44( \pm 31,7)$ & \\
$\quad$ Rural & $93( \pm 55,7)$ & $95( \pm 68,3)$ & \\
Outras cidades $(\mathrm{n})$ & $(68)$ & $(56)$ & \\
$\quad$ Urbana & $18( \pm 27,3)$ & $13( \pm 23,6)$ & $>0,64$ \\
$\quad$ Rural & $48( \pm 72,7)$ & $42( \pm 76,4)$ & \\
Período gestacional $(\mathrm{n})$ & $(235)$ & $(195)$ & $>0,85$ \\
$1^{0}$ trimestre & $37( \pm 15,8)$ & $27( \pm 13,8)$ & \\
$2^{\circ}$ trimestre & $63( \pm 26,8)$ & $54( \pm 27,7)$ & \\
$3^{\circ}$ trimestre & $135( \pm 57,4)$ & $114( \pm 58,5)$ &
\end{tabular}

(a) $\chi^{2}$; ( ) 3 casos não anotados para a variável procedência.

Como também assinalado na Tabela 1, o número de casos de malária por ambos os plasmódios aumentou proporcionalmente com o aumento do período gestacional e isto de forma altamente significativa $\left(\chi^{2}=189,97 ;\right.$ g.l. $\left.=2 ; \mathrm{p}<10^{-10}\right)$, mas não houve diferença $(p>0,85)$ entre as freqüências dos dois grupos ( $P$. vivax vs $P$. falciparum).

A média de episódios anteriores de malária foi de 2,8 $( \pm 1,7)$ no grupo P. vivax e 3,19 $( \pm 2,3)$ entre as portadoras de $P$. falciparum, não sendo a diferença significante ( $p>0,64)$, e com limites, respectivamente, de 0 a 8 e 0 a 10 episódios. No grupo vivax, a freqüência de primo-infecção foi de $5,1 \%(12 / 235)$, e no falciparum, de 10,8\% (21/195).

O tempo médio de internação foi $6,0( \pm 3,4)$ dias, com limites de 1 a 21 dias, sendo entre os casos de $P$. vivax de $5,3( \pm 2,8)$ e de $6,0( \pm 3,7)$ pelo $P$. falciparum, e essa diferença foi altamente significante $(\mathrm{p}<0,0000003)$. Durante o período de internação hospitalar, somente houve um caso de óbito $(0,2 \%$ ou $1 / 445)$, com o diagnóstico parasitológico de $P$. falciparum $(0,5 \%$ ou $1 / 195)$.

Comparando-se os dois grupos de pacientes, observa-se que (Tabela 2) houve maior proporção de grávidas com $P$. falciparum apresentando mucosas descoradas $(p<0,03)$ e icterícia $(p<0,01)$. Os registros clínicos mais freqüentes foram: febre, 97,9\% (420/429); calafrios, 71,7\% (304/424); mucosas descoradas, $67,2 \%$ (271/403); cefaléia, $62,4 \%(266 / 426)$, e icterícia, $8,2 \%(n=33 / 401)$.

Tabela 2 - Quadro clínico predominante nas pacientes, na admissão hospitalar, conforme a espécie do plasmódio.

\begin{tabular}{lccccc}
\hline & \multicolumn{5}{c}{ Plasmódio } \\
Clínica & \multicolumn{2}{c}{$\boldsymbol{P}$ vivax } & \multicolumn{2}{c}{$\boldsymbol{P}$. falciparum } & $\mathbf{p}^{\mathrm{b}}$ \\
\hline Febre & n/total $^{\mathrm{a}}$ & $\%$ & n/total $^{\mathrm{a}}$ & $\%$ & \\
Calafrios & $230 / 234$ & 98,3 & $190 / 195$ & 97,4 & $>0,73$ \\
Cefaléia & $170 / 233$ & 72,9 & $134 / 191$ & 70,2 & $>0,59$ \\
Mucosas descoradas & $139 / 234$ & 59,4 & $127 / 192$ & 66,1 & $>0,15$ \\
Icterícia & $133 / 214$ & 62,1 & $138 / 189$ & 73,0 & $<0,03$ \\
\hline
\end{tabular}

a total de casos com a informação; ${ }^{b} \chi^{2}$

Como mostra a Tabela 3, as grávidas com $P$. falciparum apresentaram maior proporção de alteração da hemoglobina $(\mathrm{p}<0,000005)$, do hematócrito $(\mathrm{p}<0,000003)$, da creatinina $(\mathrm{p}<0,003)$, da uréia $(\mathrm{p}<0,003)$, das bilirrubinas (total, $\mathrm{p}<0,0003$, e indireta, $p<0,0004)$ e da glicose $(p<0,04)$.

A terapêutica utilizada é apresentada na Tabela 4. Isoladamente, a cloroquina $(62,2 \%)$ foi o medicamento mais empregado, seguida da quinina $(17,5 \%)$, sendo desigual a distribuição $\left(\chi^{2}=11,28\right.$; $\mathrm{p}<0,03$; g.1. $=4)$ entre os usados nos casos de $P$. vivax vs. $P$. falciparum, porque foi mais freqüente o uso de cloroquina nos casos de $P$. vivax e quinina associada à clindamicina, nos casos de $P$. falciparum.

Não foi possível averiguar o curso das gestações após a alta hospitalar. Durante o período de internação hospitalar, o número de casos de abortamento foi semelhante (teste exato de Fisher, $\mathrm{p}>0,24)$ entre as grávidas com o $P$. vivax $(4 / 235$; $1,7 \%)$ e $P$. falciparum $(1 / 195 ; 0,5 \%)$; também as freqüências de casos de natimortos foram semelhantes (teste exato de Fisher, $p>0,43$ ), respectivamente, $0,4 \%(1 / 235)$ e $1,0 \%(1 / 195)$. Quanto aos nascidos vivos, em $1,2 \%(5 / 429)$ foram prematuros e outros $1,2 \%(5 / 428)$ de baixo peso, sendo respectivamente pelo $P$. falciparum 1,5\% (3/195) e $1,0 \%(2 / 192)$ dos casos. 
Tabela 3 - Resultados dos principais exames complementares à admissão hospitalar, nas grávidas com malária, distribuídos conforme a espécie do plasmódio

\section{Espécie do plasmódio}

Exames

\section{P. vivax}

\section{P. falciparum}

\begin{tabular}{|c|c|c|c|c|c|}
\hline & alterado/total & $\%$ & alterado/total & $\%$ & $p$ \\
\hline Hemoglobina $\downarrow$ & $22 / 149$ & 14,8 & $48 / 122$ & 39,3 & $<0,000005^{a}$ \\
\hline Hematócrito $\downarrow$ & $43 / 185$ & 23,2 & $73 / 153$ & 47,7 & $<0,000003^{a}$ \\
\hline Creatinina $\uparrow$ & $0 / 111$ & 0 & $8 / 102$ & 7,8 & $<0,003^{b}$ \\
\hline Bilirrubina total $\uparrow$ & $5 / 118$ & 4,4 & $22 / 110$ & 20,0 & $<0,0003^{a}$ \\
\hline Bilirrubina indireta $\uparrow$ & $5 / 119$ & 4,4 & $21 / 110$ & 19,1 & $<0,0004^{a}$ \\
\hline Glicose $\downarrow$ & $5 / 167$ & 3,0 & $12 / 141$ & 8,5 & $<0,04^{a}$ \\
\hline
\end{tabular}

${ }^{\mathrm{a}} \chi^{2 ;}$, ${ }^{\mathrm{b}}$ teste exato de Fisher.

Tabela 4 - Esquemas terapêuticos administrativos nas grávidas com malária, durante o período de internação, e distribuídos conforme a espécie do plasmódio.

\section{Espécie do plasmódio}

\begin{tabular}{|c|c|c|c|c|c|c|c|c|}
\hline \multirow[t]{2}{*}{ Esquemas terapêuticos } & \multicolumn{2}{|c|}{ P. vivax ${ }^{a}$} & \multicolumn{2}{|c|}{ P. falciparum ${ }^{b}$} & \multicolumn{2}{|c|}{ Mista } & \multicolumn{2}{|c|}{ Total } \\
\hline & $\mathrm{n}$ & $\%$ & $\mathrm{n}$ & $\%$ & $\mathrm{n}$ & $\%$ & $\mathrm{n}$ & $\%$ \\
\hline Cloroquina & 159 & 67,6 & 112 & 57,4 & 6 & 40,0 & 277 & 62,2 \\
\hline Quinina & 42 & 17,9 & 33 & 17,0 & 3 & 20,0 & 78 & 17,5 \\
\hline Cloroquina + clindamicina & 14 & 6,0 & 13 & 6,7 & 2 & 13,4 & 29 & 6,5 \\
\hline Quinina + cindamicina & 12 & 5,1 & 26 & 13,3 & 2 & 13,3 & 40 & 9,0 \\
\hline Cloroquina + quinina & 8 & 3,4 & 11 & 5,6 & 2 & 13,3 & 21 & 4,8 \\
\hline Total & 235 & 100,0 & 195 & 100,0 & 15 & 100,0 & 445 & 100 \\
\hline
\end{tabular}

$\left(\mathrm{a} v s\right.$ b): $\chi^{2}=11,28 ; p<0,03$

\section{Discussão}

Em concordância com Jarude ${ }^{16}$ e Sousa et al. ${ }^{17}$ as grávidas atendidas na Maternidade e Clínica de Mulheres Bárbara Heliodora (MCMBH) procediam em maior número da área rural da capital do Estado, município de Rio Branco, porque faltam condições de tratamento médico em serviço próximo ao local de moradia nas áreas rurais do Estado do Acre. Por sua vez, nas áreas rurais a malária tem maior endemicidade porque falta saneamento e há maior proximidade da residência ou local de trabalho com igarapés, onde também estão os principais criadouros de anofelinos ${ }^{18}$.

O perfil das grávidas estudadas foi de pacientes jovens, predominando as de idades entre 20 a 27 anos, e não houve associação da idade com a espécie do plasmódio, embora, na literatura, tenha sido descrito o risco maior de infecção pelo $P$. falciparum nas grávidas mais jovens (menores de 15 anos) ${ }^{11,19}$.

As manifestações clínicas associadas à hemólise (mucosa descorada e icterícia) foram mais freqüentes, como seria esperado, nas grávidas com malária falciparum. Porém, a tríade clínica, característica do quadro da malária (febre, calafrios e cefaléia), foi semelhante entre as grávidas com $P$. vivax e $P$. falciparum. Esses resultados têm semelhanças com os observados por outros autores ${ }^{6,10-12}$.

A síndrome anêmica, traduzida pelos valores de hemoglobina e hematócrito, foi a complicação mais freqüente e de maior intensidade encontrada no presente estudo, sendo observada em $91,6 \%$ das pacientes com malária $P$. falciparum. Achados semelhantes também foram encontrados por outros autores e em diferentes locais do mundo $^{11,13,20,21}$. Em concordância com a literatura ${ }^{6,11,12}$, também a hipoglicemia foi mais freqüente entre as grávidas infectadas com o $P$. falciparum.

O curso natural da infecção pelo plasmódio também varia conforme a intensidade de transmissão na área ${ }^{6,11,12}$. As formas graves da doença são mais freqüentes em indivíduos não expostos à infecção ou procedentes de áreas indenes ${ }^{22}$, e também estão associadas à virulência da cepa do parasito $^{23}$. Alguns autores consideram que o risco 
de desenvolver malária grave ou complicada é três vezes maior entre mulheres grávidas ${ }^{24}$, especialmente se primigestas ${ }^{11,12,25} \mathrm{e} / \mathrm{ou}$ aquelas procedentes de áreas hiperendêmica ou de transmissão instável. Nesse último caso, quando as pessoas residentes não têm imunidade prévia, estão também mais sujeitas às apresentações graves da doença ${ }^{6}$. Isto talvez explique a baixa freqüência, neste estudo, de formas graves e também de mortalidade, porque menos de $11 \%$ das grávidas (5, $1 \%$ do grupo vivax e $10,8 \%$ do falciparum) relataram infecções anteriores por Plasmodium sp.

Geralmente, tem sido demonstrado que a parasitemia é mais intensa em grávidas do que entre as não grávidas ${ }^{25}$, embora haja diferença significativa entre os sintomas da malária na mulher gestante e os observados na não gestante ${ }^{24}$. Entretanto, o nível da parasitemia não é igual durante todo o período gestacional, sendo observado aumento durante as primeiras semanas e redução mais próxima ao parto ${ }^{11,12}$.

Apesar disso, a observação, neste estudo, do maior número de pacientes no terceiro trimestre, tanto para infecções pelo $P$. vivax como pelo $P$. falciparum, não tem explicação plausível, embora Espinosa ${ }^{11}$ e Singh et al. ${ }^{21}$ também tenham encontrado resultados semelhantes, respectivamente, em Manaus e na Índia. Isto porque era esperado maior número de grávidas no primeiro trimestre da gestação, quando vários autores ${ }^{6-8,10,14,19,21,25}$ descrevem maior taxa de adoecimento. No entanto, a metodologia empregada neste estudo não fornece elementos para as mesmas conclusões.

Aparentemente, foi baixa a taxa de letalidade observada (um caso devido ao $P$. falciparum, estando a paciente no $2^{\circ}$ trimestre da gestação), mas que foi semelhante à descrita por outros estudos ${ }^{6,10,11,21}$. Isto, também, pode ter sofrido influência do menor tempo da doença, das medidas terapêuticas, dos cuidados médicos e de enfermagem, entre outras variáveis não avaliadas na presente série.

A ameaça de interrupção da gravidez, aborto e parto prematuro durante a infecção por malária têm sido descritos por diferentes autores ${ }^{10-12,20}$. Em áreas de transmissão instável de plasmódio, as perdas fetais são esperadas, explicando o aumento observado nas taxas de aborto e de natimortos durante epidemias ${ }^{6}$. A diminuição da passagem de nutrientes para o feto, devida à infecção malárica, às contrações uterinas, estimuladas pela hiperexia, e à hipóxia intra-uterina ou intraparto, secundária à sindrome anêmica, pode causar aqueles desfechos ${ }^{13}$. No entanto, é desconhecida a proporção de abortos, partos prematuros e mortes perinatais porque não há estudos, nesta região, que determinem a etiologia nesses casos por meio da busca ativa da infecção, como assinalou Espinosa ${ }^{11}$. Portanto, além da falta de informações sobre a evolução da gravidez, neste levantamento, as freqüências observadas de aborto $(1,3 \%)$, prematuridade $(1,1 \%)$ e baixo peso ao nascer $(1,1 \%)$ provavelmente estão subestimadas, sendo também inferiores às freqüências descritas por Espinosa ${ }^{11}$ e Singh et al. ${ }^{21}$. Em áreas endêmicas de malária, a diminuição no valor médio do peso do recém-nascido já foi associada à anemia, prematuridade e gestação em adolescentes ${ }^{21}$.

Entre os esquemas de tratamento antimaláricos, a cloroquina foi a mais utilizada $(62,2 \%)$, por ser a droga de primeira escolha no tratamento de grávidas ${ }^{7,11-13,20}$. No entanto, provavelmente devido à maior gravidade, nos casos com $P$. falciparum foram mais usados esquemas com dois quimioterápicos, principalmente quinina associada a clindamicina, o que é recomendado por vários autores ${ }^{6,11,12,20}$.

Em conclusão, a alta ocorrência de malária na região da cidade de Rio Branco justifica o aperfeiçoamento do programa de diagnóstico e tratamento na rede pública de saúde, especialmente nos serviços voltados à assistência pré-natal e de perinatalogia, porque essa infecção continua sendo relevante fator de morbidade entre as grávidas e tem efeitos ainda pouco investigados sobre a saúde da mulher e do recém-nascido.

\section{ABSTRACT}

Purpose: to describe the frequency of clinical and laboratorial findings in pregnant women with malaria.

Methods: a descriptive study was performed including 445 pregnant women with a positive diagnosis of malaria of the Maternity and women's Clinic Bárbara Heliodora (Rio Branco, Acre State), from January 1996 to December 2001. These cases were reviewed and the pregantwomen with malaria were selected. Results: a total of 33,420 patients were hospitalized in that period. Of these, 445 pregnant women (1.4\%) were included. Among these, the frequency of the infection with Plasmodium vivax was $52.8 \%$ ( $n=235)$, with $\mathrm{P}$. falciparum, $43.8 \%(n=195)$, and $3.4 \%(n=15)$ with both plasmodia. The most frequent clinical and laboratorial alterations $(p<0.05)$ were observed in the patients with $\mathrm{P}$. falciparum: pale mucosa, jaundice hemoglobin and hematocrit decrease, hypoglycemia andincrease in serum aminotransferase, urea, creatinine and in bilirubin levels. Only one patient (1/445), a pregnant woman infected with $\mathrm{P}$. falciparum died. Abortions, premature birth and low birth weight were detected in 1.3, 1.1 and $1.1 \%$ of the cases, respectively. Conclusions: these findings reflect the repercussion of malaria during pregnancy, and justify the existence of qualified medical care in obstetric units for diagnosis and treatment of these cases, especially in the Amazon region.

KEYWORDS: Malaria. Pregnancy complications. Infectious diseases in pregnancy. Anemia. 


\section{Referências}

1. Word Health Organization. Malaria. Revised Fact Sheet $n^{\circ}$ 94, 2001 [cited 2002 Mar 23]. Available from: http//www.who.int

2.Ministério da Saúde. Malária: aspectos epidemiológicos. 1999-2000 [acesso 14 abr 2002]. Disponivel em: http://www.funasa.gov.br/guiaepi/doenças / malaria

3. Ministério de Saúde. Situação atual da doença: relatórios gerenciais. Brasília: Fundação Nacional de Saúde; 2002. p.1-3.

4. Souza JM, Couto AARC, Silva EB, Abdon NP, Silva RSU. Malária. In: Leão RNQ, editor. Doenças Infecciosas e Parasitárias: enfoque amazônico. $1^{\text {a }}$ ed. Belém: CEJUP/UEPA; 1997. p. 645-69.

5. Martins FSV, Ramos Filho CF, Santos GCT. Malária. In: Schechter M, editor. Doenças Infecciosas: conduta diagnóstica e terapêutica. $2^{a}$ ed. Rio de Janeiro: Guanabara Koogan; 1998. p.182-96.

6. Menendez C. Malaria during pregnancy: a priority area of malaria research and control. Parasitol Today $1995 ; 11: 178-82$.

7. Rogerson SJ, Brown GV. Chondroitin sulphate A as an adherence receptor for Plasmodium falciparuminfected erythrocytes. Parasitol Today 1997; 13:70-5.

8. Beeson JG, Cooke BM, Rowe JA, Rogerson SJ. Expanding the paradigms of placental malaria. Trends Parasitol 2002; 18:145-7.

9. Lekana Douki JB, Traore B, Costa FT, et al. Sequestration of Plasmodium falciparum-infected erythrocytes to chondroitin sulfate $A$, a receptor for maternal malaria: monoclonal antibodies against the native parasite ligand reveal panreactive epitopes in placental isolates. Blood 2002; 100:1478-83.

10.Sandbu S, Nokleby H. Young children, pregnant women and travelling abroad. Tidsskr Nor Laegeforen 2002; 122:1573-6.

11.Espinosa FM. Malária na gravidez: estudo de pacientes do Instituto de Medicina Tropical do Amazonas, Brasil, 1990-1997 [dissertação]. Rio de Janeiro: Fiocruz; 1998.

12.Alecrim WD, Espinosa FE, Alecrim MG. Plasmodium falciparum infection in the pregnant patient. Infect Dis Clin North Am 2000; 14:83-95.
13. Nussenblatt TV, Semba RD. Micronutrient malnutrition and the pathogenesis of malaria anemia. Acta Trop 2002; 82:321-37.

14. Menendez C, Fleming AF, Alonso PL. Malaria-related anaemia. Parasitol Today 2000; 16:469-76.

15.Instituto Brasileiro de Geografia e Estatística. O Brasil município por município [acesso $07 \mathrm{dez}$ 2002]. Disponivel em: http//www.ibge.gov.br/home/ estatística/população/Cidadesat/

16.Jarude R. Malária em gestantes de uma maternidade pública de Rio Branco, Acre, Brasil, 1996-2001 [dissertação]. Salvador: Universidade Federal da Bahia; 2002.

17. Sousa HP, Sabóia RC, Franco SV. A incidência de malária em gestantes numa maternidade estadual de Rio Branco - Acre [monografia]. Rio Branco: Universidade Federal do Acre; 2000.

18.Suarez MMC. Estudo do processo de transmissão da malária em uma área de invasão recente na cidade Manaus - Amazonas [dissertação]. Rio de Janeiro: Fiocruz; 1977.

19.Mutabingwa TK, Malle LN, de Geus A, Oosting J. Malaria chemosuppression in pregnancy. II. Its effect on maternal haemoglobin levels, placental malaria and birth weight. Trop Geogr Med 1993; 45:49-55.

20.Ahmed SM, Abd Al-Rhim SK, Mohamedani AA, Habour AB, Sadek AA. Malaria parasitemia during delivery. Saudi Med J 2002; 23:684-8.

21.Singh N, Mehra RK, Srivastava N. Malaria during pregnancy and infancy, in an area of intense malaria transmission in central India. Ann Trop Med Parasitol 2001; 95:19-29.

22.Baird JK. Host age a determinant of naturally acquired-immunity to Plasmodium falciparum. Parasitol Today 1995; 11:105-11.

23.Gupta S, Hill AV, Kwiatkowski D, Greenwood AM, Greenwood BM, Day KP. Parasite virulence and disease patterns in Plasmodium falciparum malaria. Proc Natl Acad Sci USA 1994; 91:3715-9.

24.Luxemburger C, Ricci F, Nosten F, Raimond D, Bathet S, White NJ. The epidemiology of severe malaria in an area of low transmission in Thailand. Trans R Soc Trop Med Hyg 1997; 91:256-62.

25.Duffy P, Fried M. Malaria in pregnancy. Bull World Health Organization [serial online] 2002 [cited 2002 Jul 10]; 80(5). Available from: http://www.who.int/ bulletin/pdf/2002/bul-5-E-2002/80(5)418.pdf 